\title{
(Mis)interpreting Mathematical Models: Drift as a Physical Process
}

\author{
Roberta L. Millstein, ${ }^{\S}$ Robert A. Skipper, $\mathcal{F r}^{\ddagger}$ and Michael R. Dietrich $₫$
}

\begin{abstract}
Recently, a number of philosophers of biology (e.g., Matthen and Ariew 2002; Walsh, Lewens, and Ariew 2002; Pigliucci and Kaplan 2006; Walsh 2007) have endorsed views about random drift that, we will argue, rest on an implicit assumption that the meaning of concepts such as drift can be understood through an examination of the mathematical models in which drift appears. They also seem to implicitly assume that ontological questions about the causality (or lack thereof) of terms appearing in the models can be gleaned from the models alone. We will question these general assumptions by showing how the same equation - the simple $(p+q)^{2}=p^{2}+2 p q+q^{2}-$ can be given radically different interpretations, one of which is a physical, causal process and one of which is not. This shows that mathematical models on their own yield neither interpretations nor ontological conclusions. Instead, we argue that these issues can only be resolved by considering the phenomena that the models were originally designed to represent and the phenomena to which the models are currently applied. When one does take those factors into account, starting with the motivation for Sewall Wright's and R.A. Fisher's early drift models and ending with contemporary applications, a very different picture of the concept of drift emerges. On this view, drift is a term for a set of physical processes, namely, indiscriminate sampling processes (Beatty 1984; Hodge 1987; Millstein 2002, 2005).
\end{abstract}

\section{KEYWORDS}

Indiscriminate sampling outcome $\bullet$ James Crow $\bullet$ Mathematical models $\bullet$ Motoo Kimura $\bullet$ Population genetics $\bullet$ Process $\bullet$ R. A. Fisher $\bullet$ Random genetic drift $\bullet$ Sewall Wright

\section{Introduction}

Consider the following account of drift:

Drift, in any of its forms, is a statistical property of an ensemble of trials or events: drift is statistical error. A series of births, survivals, deaths, and reproductions manifests drift just if the outcomemeasured as changes in trait frequencies - diverges from that predicted by differences in fitness (Walsh, Lewens, and Ariew 2002: 459).

This defines drift purely in terms of its outcome, namely, in terms of the deviation of the actual outcome from fitness expectations. In other words, if in a given generation the proportions of types in a population exactly

$\S$ Department of Philosophy, University of California-Davis, Davis, CA 95616

$\ddagger$ Department of Philosophy and Center for Environmental Studies, University of Cincinnati

- Department of Biological Sciences, Dartmouth College

E-mail: RLMillstein@ucdavis.edu

Received 18 April 2009; Accepted 19 August 2009 
match those expected from the fitness values of the different types in the population, then on this view, there is no drift. Of course, there will usually be some deviation, and thus some drift, given that all real populations are finite. But that's just a mathematical consequence, in the same way that one may flip a fair coin ten times without obtaining half heads. Thus on this view, nothing physical is happening here; there is no physical process of drift. We shall call this the Drift as Outcome Alone (DOA) definition. Others works endorse DOA; see, e.g., Matthen and Ariew (2002); Pigliucci and Kaplan (2006); and Walsh (2007). Plutynski (2007) is sympathetic, but falls short of endorsement.

On our view, the DOA conception of drift rests on an implicit assumption that the concept of drift is to be understood solely through an examination of the mathematical models in which drift appears. Indeed, the DOA view seems to assume that questions about the causality (or lack thereof) of drift can be gleaned from the models alone. We think this view is mistaken. We will argue that an examination of the mathematical models of drift alone cannot yield a proper understanding of drift because: 1) the same mathematical model can give rise to more than one interpretation; moreover, there are different drift models to consider, and 2) a proper interpretation of drift is informed by historical and contemporary biological practice; DOA is not.

Ultimately, correctly interpreting mathematical models of drift by relating the models to the physical systems being modeled results in a conception of drift as an indiscriminate sampling process (Beatty 1984), i.e., a physical process where heritable physical differences between entities are causally irrelevant to differences in reproductive success (Millstein 2002, 2005; see also Hodge 1987). Entities that can be indiscriminately sampled include, but are not limited to, "parents" (i.e., organisms) and gametes; Beatty (1984) dubs the former "indiscriminate parent sampling" and the latter "indiscriminate gamete sampling." Or so we will argue. We begin with a very brief review of common conceptualizations of drift among biologists and philosophers.

\section{Common conceptualizations of drift among biologists and philosophers}

DOA is not the most common conceptualization of drift among biologists; biologists typically describe drift as an indiscriminate sampling process leading to a certain type of outcome. That is, biologists typically define drift not as outcome alone, but rather, process and outcome together, as the following examples illustrate (Hartl and Clark (1996) may be an exception here; they seem to describe drift as a process alone). The indiscriminate sampling process that is described most often is indiscriminate gamete sampling, although other biological phenomena such as indiscriminate parent sampling, indiscriminate founding events (also known as the "founder effect" or "founder principle"), and indiscriminate bottleneck events are also often described as forms of drift (all of which are forms of indiscriminate sampling).

At first glance, Futuyma provides a description of drift that sounds similar to the DOA account: he says that drift occurs as a consequence of small population size and compares drift to tossing a coin a small number of times (2005: 196), as DOA proponents do. However, in his chapter focusing on drift, Futuyma makes it clear that drift involves indiscriminate gamete sampling:

...the frequency of an allele can change...because one or few copies of the... allele may happen not to be included in those gametes that unite into zygotes, or may happen not to be carried by the offspring that survive to reproductive age. The genes included in any generation, whether in newly formed zygotes or in offspring that survive to reproduce, are a sample of the genes carried by the previous generation. Any sample is subject to random variation, or sampling error (2005: 227).

In the subsequent paragraph, Futuyma invokes indiscriminate parent sampling by giving an example where changes in gene frequency in a population of snails are the result of being squished by cows, a process in which the color of the snails is causally irrelevant.

Turning to other popular evolution textbooks, Roughgarden similarly explains indiscriminate gamete sampling in a finite population before asserting, "Genetic drift is the name for changes in gene frequency caused by this sampling error" (1996: 57-58). And according to Ridley's discussion of drift: 
...random changes in gene frequencies between generations are called genetic drift, random drift, or (simply) drift...Genetic drift is not confined to cases of selective neutrality. When selection is acting at a locus, random sampling (1) also influences the change in gene frequencies between generations... The sampling of gametes is only the first stage at which random sampling occurs. It continues at every stage as the adult population of a new generation grows up (2003: 138-139).

Thus, for all three of these biologists, drift is indeed partially characterized as an outcome (a change in gene frequencies), but it is an outcome caused by a certain type of physical process (indiscriminate parent or gamete sampling) - not as outcome alone.

Although it is not our target here, it can be argued that problems arise for accounts of drift which incorporate both process and outcome, as these biologists' definitions do; to mention one problem, authors usually end up vacillating between drift-as-process and drift-as-outcome, leading to inconsistencies (as Millstein 2002, 2005 has argued). Even if it were the common definition among biologists, such definitions should not be accepted uncritically. There is a role for philosophical analysis in order to achieve, e.g., consistency, clarity, and representation of distinct phenomena. Indeed, we write in the spirit of Mills and Beatty (1979), who begin with biologists' definitions of fitness but re-evaluate those definitions in light of biological practice (and so honor the implicit use rather than the explicit definition; the use of the term "drift" will be discussed further below). Regardless, it seems clear that standard conceptions of drift articulated by biologists are not consistent with the DOA view, since they incorporate drift-as-process.

DOA may in fact be the most common definition of drift among philosophers. For example, Pigliucci and Kaplan state:

In a case in which the changes observed are close to the changes statistically expected for relevantly similar populations, we might say that the outcome reflects our expectations from predictive selection; in a case in which the changes are more distant from the mean, we might say that the outcome does not reflect those expectations - that is, we might choose to call it an example of drift. But that does not imply that any kind of process took place in the latter population that did not take place in the former... It is worth stressing the conclusion that drift is not a process in any meaningful sense (Pigliucci and Kaplan 2006: 28-29).

Thus, Pigliucci and Kaplan have seemingly (2) endorsed DOA: drift is not a process "in any meaningful sense," but rather, a certain type of outcome (namely, an outcome that deviates from the expectations of selection).

However, not all philosophers endorse DOA; some philosophers endorse a combined process/outcome definition and some endorse our view of drift as a process. For example, Richardson describes drift in terms of its outcome alone: " $[\mathrm{g}]$ enetic drift is sometimes described as the 'error' in the transmission of types from generation to generation, arising from finite population size" (2006: 643). However, Richardson is not committed to the view that drift is purely mathematical, as the proponents of DOA are, since he characterizes drift as causal. Brandon, on the other hand, defines drift as "any deviation from the expected levels of reproduction due to sampling error" (2005: 169). He thinks that it "is surely right to think of selection and drift as processes" but that "the outcomes are necessary not only to operationalize the distinction, but also to make sense of it conceptually" (2005: 169). So, Brandon's view is similar to those of the biologists described previously, except that he believes "there is a single process, sampling" (2005: 167).

But just as one should not uncritically accept standard, textbook conceptions of drift, one should not uncritically accept philosophers' conceptions either. There should be some connection to biologists' definitions, even if those definitions are not fully authoritative. The general point is that any definition, whether it derives from a biologist or a philosopher, must be critically examined and defended. With that said, it's clear that DOA can't be defended either as the most common definition among contemporary biologists (because it isn't) or as the most common definition among philosophers (it might be, but that doesn't settle the issue without substantive connection to biological practice). As we argue in what follows, biological practice doesn't bear out the DOA view. But first, let's consider how DOA might be defended. 


\section{Examination of the mathematical models of drift alone}

Since DOA cannot be justified by an appeal to common conceptions among biologists and philosophers, the most charitable interpretation is that DOA is in accord with the mathematical models of drift, although it cannot be derived entirely consistently (see Millstein and Skipper 2007 for discussion). But setting concerns about consistency aside, one might think DOA is the most natural understanding of drift based on mathematical presentations of drift models in some population genetics textbooks. Such presentations begin by specifying the conditions under which gene frequencies remain unchanged from one generation to the next, via the highly idealized Hardy-Weinberg Principle. Then, one by one, the assumptions underlying the Hardy-Weinberg principle are relaxed, e.g., by incorporating fitness differences (and thus selection), and by introducing finite population size. Drift seems to be introduced when finite population size is introduced, and so, one might think that drift is merely the effect of finite population size, and thus, purely mathematical. (This is what we mean when we say that DOA proponents seem to understand drift solely through an examination of the mathematical models in which drift appears). If this reading of population genetics textbooks is right, (3) would it justify DOA?

We contend that it does not - that mathematics cannot be the sole basis for interpretation because the same equation can be given radically different interpretations, one physical and one not. To see this, consider the equation $(p+q)^{2}=p^{2}+2 p q+q^{2}=1$.

This equation could be understood as the Hardy-Weinberg Principle itself. Under this interpretation, the equation represents an infinite (or very large) sexually reproducing population of diploid organisms undergoing random mating; we focus on one locus with two alleles, $\mathrm{A}$ and $\mathrm{a}$, the former which has a frequency $\mathrm{p}$ and the latter which has a frequency $\mathrm{q}$. The values $\mathrm{p}^{2}, 2 \mathrm{pq}$, and $\mathrm{q}^{2}$ represent the proportions that AA, Aa, and aa will maintain (respectively) in the population from generation to generation, assuming that the idealizing assumptions hold.

Now consider that the same equation, $(p+q)^{2}=p^{2}+2 p q+q^{2}=1$, can have a very different interpretation. Suppose we have a square with sides of length $p+q$, as follows:

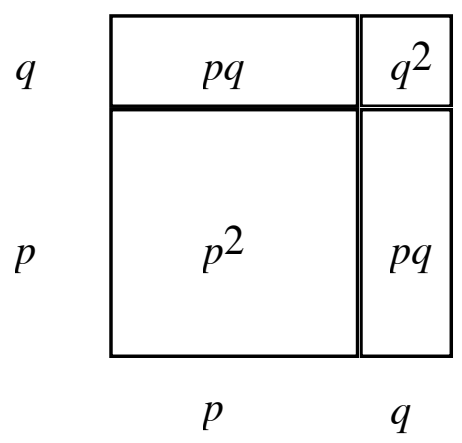

There are two ways to calculate the area of the large square. Most directly, its area is $(p+q)^{2}$. But its area can also be calculated as the sum of the areas of the four rectangles: $\mathrm{p}^{2}+2 \mathrm{pq}+\mathrm{q}^{2}$. And since any length can be designated as 1 unit, we can say that the area of the new square is $(p+q)^{2}=p^{2}+2 p q+q^{2}=1$; the same equation that is used for the Hardy-Weinberg Principle. Let us call this the "sum of areas" interpretation.

As the Hardy-Weinberg Principle, the equation represents a physical process: the maintenance of genotype frequencies in a population of randomly mating, sexually reproducing organisms from one generation to the next. The physical process can be represented mathematically, but it is not purely mathematical. As the sum of areas, however, the equation does not represent a physical process. It is purely mathematical; it is simply a geometrical relationship. Thus, it is a mistake to derive definitions from mathematics alone, as DOA seems to do, since many, very different definitions can be derived from the same equation. Moreover, it is problematic to think that ontological questions about the causality (or lack thereof) of terms appearing in equations can be gleaned from the equations alone. On some interpretations, a physical process is represented, but on others, it is not. There is no way to tell from the mathematics alone. 
So, one reason that it is a mistake to think that we can glean definitions of drift from mathematics alone, as DOA seems to do, is that the same mathematics can give rise to radically different interpretations.

This can be recast in more philosophical terms using the semantic approach to theories. Although the semantic approach developed its analysis of models and their interpretation to address the problem of the constitution and structure of theory in science, their account of models provides a sophisticated framework for understanding exactly the kinds of mathematical population genetics models used to represent drift (Lloyd 1988). According to the semantic approach, a model is a non-linguistic entity that can be represented in terms of variables and rules or laws describing the functions operating on those variables. The state of the model refers to any particular set of values for the variables in that model. The collection of all of the states of the model composes the model's state space. The rules of a theory describe the possible relationships of coexistence, interaction, and temporal succession of the variables in the model. In doing so they describe trajectories through the state space for that model (Lloyd 1988: 19). One virtue claimed for this approach to understanding models and theories is that it coheres strongly with the ways in which biologists understand models with biology (Lloyd 1988, Lewontin 1963).

Models, according to the semantic approach, are always ideal structures. Interpreting a model, then, involves proposing that certain features of the model, some or all of variables and functions, correspond to certain features of the world. This claim of empirical correspondence is then subject to verification and refutation. So, in terms of the binomial equation, the equation represents a mathematical model with two variables and a function describing their interaction. One interpretation of this equation involves the claim that $\mathrm{p}$ and $\mathrm{q}$ correspond to the frequencies of two alleles at a locus; the other interpretation involes the claim that $\mathrm{p}$ and $\mathrm{q}$ correspond to lengths of the side of a square. (Of course, there are other possible interpretations). The first interpretation is justified if the model provides an empirically adequate description of the dynamic behavior of two alleles at a locus and if the assumptions made in the model's interpretation are themselves justified (Lloyd 1988, Skipper 2000, Dietrich and Skipper 2008). (4) The justification of the model's interpretation depends crucially, we claim, on claims about the physical processes that affect the allele frequencies and their dynamics. The second interpretation of the equation involves claims about geometric and mathematical relationships that can be justified by appeal to geometric proof, as opposed to physical correspondence. The equation alone or the model alone does not carry with it any particular interpretation, although it may constrain which interpretations are possible given the variables and their interaction. Claims about drift are claims about empirically justified interpretations of these models. These justification depend crucially on our understanding of underlying physical processes.

Another reason it is a mistake to think that we can glean definitions of drift from mathematics alone is that there isn't just one mathematical model of drift. Rather, there are many different models, each with its own mathematical representation and each with its own biological justification, as the following discussion shows. None are purely mathematical.

Philosophers of biology often start discussions of drift with the simple Wright-Fisher model, the core of which is the binomial distribution. Here, there are assumed to be $\mathrm{N}$ diploid adults in a population, mating randomly, with a gene that has a frequency of $\mathrm{p}_{0}$. Adults make an infinite number of gametes having the same allele frequency. From the pool, $2 \mathrm{~N}$ gametes are drawn at random to constitute the $\mathrm{N}$ diploid individuals for the next generation. The binomial distribution is key. The probability that i A alleles make it to the next generation is the binomial probability, $\operatorname{Prob}\{\mathrm{i}\}=\left[\left(2 \mathrm{~N} ! / \mathrm{i} !(2 \mathrm{~N}=\mathrm{i}) ! \mathrm{p}^{\mathrm{i}} \mathrm{q}^{2 \mathrm{~N}-\mathrm{i}}\right.\right.$, where i may be $0,1, \ldots$, $2 \mathrm{~N}, \mathrm{~N}$ is the population size, and $\mathrm{p}$ and $\mathrm{q}$ are allele frequencies (and $\mathrm{q}=1-\mathrm{p}$ ). The binomial distribution describes the probability of $\mathrm{i}$ events of copying allele $\mathrm{A}$, where the probability of copying an $\mathrm{A}$ allele is $\mathrm{p}$ over $\mathrm{n}=2 \mathrm{~N}$ (in this case) independent trials.

The Wright-Fisher model is a simple and elegant model of the central statistical features of drift, i.e., the mean and variance of $\mathrm{p}^{\prime}$ and the change in heterozygosity, H. But, as John Gillespie (2004: 49) has aptly pointed out: "its biological underpinnings can make us queasy." (5) He continues, "populations do not reproduce by calling in their local statistician and asking her to pick exactly $2 \mathrm{~N}$ gametes at random (with replacement) and toss them into the next generation" (p. 49). Differently put, drift (the physical process) is not a binomial sampling process.

There are other ways of mathematically modeling drift. Since we have already invoked Gillespie, consider his general model of drift (2004: 49-51). Assume a diploid population of N members and a two- 
allele locus, $\mathrm{A} 1$ and $\mathrm{A} 2$ at frequencies $\mathrm{p}$ and $\mathrm{q}=(1-\mathrm{p})$. Say the population mates at random. In this case, each of the $2 \mathrm{~Np} \mathrm{Al}$ gametes will make up the next generation with a random number of offspring gametes. Let the random variable $\mathrm{Xi}$ represent the number of offspring gametes from the ith $\mathrm{Al}$ gamete and $\mathrm{Yi}$ represents the number from the ith $\mathrm{Al}$ gamete. $\mathrm{Xi}$ and $\mathrm{Yi}$ may take values $0,1,2$, and so on. The total number of $\mathrm{A} 1$ alleles in the next generation may be written as the random variable $\mathrm{X}=\mathrm{X} 1+\mathrm{X} 2+\ldots+$ $\mathrm{X} 2 \mathrm{NP}$. Similarly for $\mathrm{Y}$. The allele frequency in the next generation is the random variable $\mathrm{p}^{\prime}=\mathrm{X} / \mathrm{X}+\mathrm{Y}$, i.e., the number of $\mathrm{Al}$ gametes in the next generation divided by the total number of gametes. There is no restriction on the distribution of the numbers of offspring nor on the total number of offspring gametes.

Gillespie's generalized model, like the Wright-Fisher model, captures the core statistical features of drift, the mean and variance of $\mathrm{p}^{\prime}$ and the change in heterozygosity, $\mathrm{H}$. The difference is that the generalized model is not tied to binomial sampling. Rather than a population calling in the local statistician, Gillespie's generalized model reflects, in a biologically more realistic way than the Wright-Fisher model, that "individuals find mates, have babies, babies survive to reproduce, etc." (p. 49).

Our point here is this: There are numerous mathematical models of drift. Taking any one model as central to understanding drift takes us in one of many interpretive directions. The Wright-Fisher model sees drift as a binomial sampling process, whereas Gillespie's general model is not so constrained. If we consider as well other models of drift, such as the Moran model, the Cannings model, or the coalescent, then other interpretations are sure to result. The varying interpretive directions are guided mainly by the assumptions embedded in the model. For example, the Wright-Fisher model assumes that all the alleles at each locus disaggregate and are sampled randomly. This central assumption of the model belies the fact that alleles are linked together in chromosomes. In fact, taking such linkage into account might, as it did Gillespie, lead one away from drift as an explanation of decreases in heterozygosity and toward random effects of linked selection (Skipper 2006).

It is not clear whether there is any particular reason to choose one drift model over another in trying to understand the concept of drift, though there may be other reasons to choose a particular model (e.g., tractability). All mathematical models of drift make simplifying assumptions that belie biological reality; a biologically realistic model is surely practically impossible. So, conceptually reifying simplistic models will either lead to strange claims about what sort of process drift is (e.g., binomial sampling) or false claims that all there is to understanding drift is outcomes. More specifically, DOA treats drift and flips of a coin as though they were perfectly analogous. However, that is only true on the Wright-Fisher model, where drift is modeled as binomial sampling. And the motivations behind the alternative models are baffling for the DOA view. Gillespie, for example, is seeking a model with more realistic biological assumptions about drift; this makes no sense if drift were only a statistical outcome.

None of this is to say that examining mathematical models of drift plays no role in our understanding of what drift is. Rather, it is to say that models themselves are not the ultimate resource. The interpretation of models is a matter of synthesizing theoretical, data, and experimental models. That is, interpreting models really means bringing in assumptions about the system that is being modeled. After all, models are, as Giere (1988) so clearly points out, built as representations of specific aspects of physical systems. In that sense, interpreting models means making the assumptions about how the model is constructed plain. Indeed, interpretation isn't a matter of deriving the properties of a modeled system from the model. To be sure, we want our population genetics models to give us measurements we can use - the mean and variance of $\mathrm{p}$ ', for example. But those measurements don't then exhaust the system being modeled. What we are measuring and how we think we can do so with some specific model must be spelled out. And that invariably involves bringing in metaphysical (causal) assumptions (among others) about the real world system being modeled. The process of relating models to data (through an examination of theoretical, data, and experimental models) is then meant to bear out the (homomorphic) relationship between model and real world systems. As we will argue in the next section, when we model drift, we model indiscriminate sampling processes and, so, a proper conception of drift requires a consideration of those processes. 


\section{Consideration of historical and contemporary biological practice}

Examination of the first articulations of a term allow us to answer the questions, "What was the original purpose of this term? What was its role in theory and in practice? In particular, was the term intended to represent a physical phenomenon or was its role purely mathematical?"

The first mentions of "drift" can be traced to Sewall Wright and R. A. Fisher, although neither claimed to have developed the idea behind drift; Fisher first discussed the idea in his discussion of the writings of A.C. and A. L. Hagedoorn (1921), whereas Wright (1951) credits Gulick (1872) rather than the Hagedoorns (see Beatty 1992 for discussion). Moreover, it is fair to say that Wright and Fisher are jointly responsible for the first mathematical models of drift in population genetics. (6) Thus, they are the central figures to consider in deciding whether drift was intended to be purely mathematical or representative of a physical process.

What the following three quotes will show is that Fisher and Wright indeed intended for "drift" to represent a physical process. More specifically, they saw drift as an indiscriminate sampling process (as described earlier).

(i). Fisher (1921) invokes drift as indiscriminate parent sampling. Note also that the phenomenon itself is taken to occur regardless of the size of the population, although the speed of its effect varies with the population size:

[The Hagedoorns] believe that the random selection of individuals to become the parents of the next generation is a more important factor in reducing the variability of a species than is the natural selection of advantageous characters. The whole question is worthy of a thorough discussion, but the authors evidently lack the statistical knowledge necessary for its adequate treatment. By taking simple examples of species of only two or three individuals it is easy to show that by random selection the variability will be rapidly reduced. It by no means follows that the reduction will be equally rapid in an interbreeding group of 2 or 3 million individuals (pp. 467-8; emphasis in original).

(ii). Wright (1932) describes drift as both indiscriminate parent sampling and indiscriminate gamete sampling; again, the physical phenomenon is seen as producing a certain kind of effect (a change in gene frequency from one generation to the next):

If the population is not indefinitely large, another factor must be taken into account: the effects of accidents of sampling among those that survive and become parents in each generation and among the germ cells of these...Gene frequency in a given generation is in general a little different one way or the other from that in the preceding, merely by chance (p. 360)

(iii). Fisher and Ford (1947) discuss drift as indiscriminate gamete sampling; once again, the strength of the effect (but not the occurrence of the phenomenon) depends on population size:

Great evolutionary importance has been attached by Sewell [sic] Wright $(1931,1932,1935,1940)$ to the fact that small shifts in the gene-ratios of all segregating factors will occur from generation to generation owing to the errors of random sampling in the process by which the gametes available in any one generation are chosen to constitute the next. Such chance deviations will, of course, be greater the smaller the isolated population concerned (p. 167).

These quotes are only three among the many that we could display; see also, e.g., Fisher (1922ab) and Wright $(1931,1937,1948)$. All three point to the same conclusion: the biologists who developed the mathematical models of drift did so with the intention of modeling physical processes (the indiscriminate sampling processes) that they took to be occurring in nature. It might be argued that, as with the definitions of contemporary biologists, Fisher's and Wright's definitions invoke both process and outcome; again, we would suggest that such definitions not be accepted uncritically. But even if the best definition of drift were to combine process with outcome, it would 
still provide no support for DOA and the claim that drift is purely mathematical. The drift models were not intended simply to incorporate the mathematical consequences of finite population size, as the proponents of DOA would have us believe; population size does have a role to play, but only in determining the strength and speed of the effect of the indiscriminate sampling processes being modeled.

Just to be clear, it is not our contention that Fisher and Wright consistently used the term "drift" to represent indiscriminate sampling processes. Wright in particular became quite expansive in the use of the term, sometimes conflating it with inbreeding, a distinct biological phenomenon that is also connected to small population size. Another notorious expansion was to consider fluctuations in mutation rate, fluctuations in migration, and fluctuations in selection to be drift (Wright 1955). In response, Cain and Currey (1963) justifiably noted " $[\mathrm{w}]$ hatever may be the conveniences for mathematical geneticists of considering together all processes regarded as random, irrespective of their biological significance, the worker on actual examples must classify processes according to their biological significance" (Cain and Currey 1963: 59). Furthermore, they asserted, "in practice the lumping of 'random' processes prevents the proper analysis of actual situations...Such confusions can only lead to erroneous general conclusions about the relative importance of selection, sampling error, mutation and other factors" (Cain and Currey 1963: 59). Because "[s]ampling error is biologically a very different phenomenon from selection, and as Wright has declared that 'genetic drift' must have a wider connotation, we propose to refer to the effects of sampling error as sampling drift" (Cain and Currey 1963: 59). Wright appears to have agreed, at least in part; for example, he uses the term "sampling drift" in his 1978 magnum opus, Evolution and the Genetics of Populations. In short, although we can understand the reasons why Wright expanded his use of the term drift fluctuations in selection, mutation, and migration could play the same role as indiscriminate sampling in Wright's shifting balance model, and that was his primary concern - that does not imply that the expansion was justified. In any case, note that even as an expanded term, drift still represents physical (biological) processes, not merely mathematical constructs as DOA proponents contend.

Nor is it our contention that "drift represents biological processes just because Wright and Fisher said that they did," although we are claiming the first uses of a term are relevant to its understanding. We are also claiming that Wright and Fisher had good reason to think that there were biological processes in nature that needed to be represented in drift models in order to better track evolutionary changes in a population.

Plutynski (2007) has suggested that the history of the use of the term "drift" is too varied (she calls it a "catchall term") to draw any definitive philosophical conclusions about how it ought to be used. We don't have any substantive disagreements with her presentations of the views of Wright, Fisher, et al., but we think she has overstated the variety of meanings. It is true that drift has been used to describe processes at different levels of organization (gametes and organisms, for example), (7) but many of these can be construed as indiscriminate sampling. That is, indiscriminate gamete sampling and indiscriminate organism (or "parent") sampling are both forms of drift. We contend (taking our historians' hats off for a moment and putting our philosophers' hats on) that those uses that cannot be construed as indiscriminate sampling describe different biological processes and thus ought to be referred to by different terms, as Cain and Currey suggested.

One might object that we are interpreting Fisher and Wright incorrectly. But we don't think they would make such a claim. In fact, we think their own understanding of the relationship between their quantitative and qualitative evolutionary theories is just more evidence not only that our interpretations are correct, but that they took themselves to be arguing over the processes of evolution - especially drift and its role among them - as opposed to the models themselves.

In 1930, Fisher's The Genetical Theory of Natural Selection was published, and Wright had submitted for review his famous 1931 paper, "Evolution in Mendelian Populations," to Genetics. Wright was also invited to write a review of Fisher's book for The Journal of Heredity. During the months of 1930, Fisher and Wright corresponded about the details of their quantitative theories. Each was concerned to bring their alternative mathematical approaches into agreement. By October, they managed to do so. But that reconciliation did nothing to quell what Fisher and Wright considered their more substantive disagreement, which was over their qualitative evolutionary theories. On October 15, 1930, Wright wrote: 
...there is now no mathematical difference between our results in the cases which can be compared. I have discussed [in the review of Fisher's book] at some length the rather different interpretations of the role of selection which we have reached and will be much interested in getting your criticism of my view. (Wright to Fisher, October 15, 1930, in Provine 1986: 265).

The "rather different interpretations" that Wright is talking about concern, mainly, the implications for the role of certain evolutionary processes given his and Fisher's alternative assumptions about population size. According to Wright, Fisher's assumption that populations are large enough to ignore "the effects of random sampling of gametes" leads to a too-slow evolutionary process. That is, selection and mutation alone are too slow in geologic time to yield the cumulative evolution observed. Wright famously believed that a global population subdivided into semi-isolated, intermediate-sized populations would yield a correct evolutionary tempo because indiscriminate gamete sampling would increase the amount of genetic variation on which selection could act and migration would transport favorable adaptations through the global population via migration (Wright 1930: 354).

Wright took pains in his 1931 paper to make plain the biological weaknesses of the quantitative approach to evolution. Indeed, he devoted an entire section of his 1931 paper to laying out and justifying his qualitative interpretation of his quantitative views, clarifying what he took to be the "factors" of genetic homogeneity and heterogeneity in evolution and then analyzed their roles and effects qualitatively. Perhaps his most famous such qualitative discussion of his evolutionary theory is in his 1932 adaptive landscape paper, in which he is concerned to articulate the "mechanism by which the species may continually find its way from lower to higher" adaptive peaks on the multidimensional surface of selective value. Drift -"a trial and error mechanism" — was key to this articulation (Wright 1932: 359).

Wright and Fisher famously disagreed about, among other things, the role of drift in the evolutionary process. But we think it's fair to say that Fisher agreed with Wright that relating their quantitative models to their qualitative views of the evolutionary process was crucial to understanding evolution. In response to Wright's October 15, 1930 letter, Fisher wrote:

Mathematicians always tend to assume that the hardest mathematics will be the most important, and this is perhaps true enough in the well worn topics. It is certainly not true of my [1930] book, where the apparently non-mathematically parts, where I have left the mathematics undone, are often of the greatest ultimate interest. (Fisher to Wright, October 25, 1930, in Provine 1986: 266, emphasis in original).

Comparatively, Wright was much more concerned with making the differences between his quantitative and qualitative evolutionary theories clear in print than Fisher. But Fisher's letter to Wright is certainly useful for sorting out how to approach conceptual questions in population genetics. Working through all that makes up the controversies between Fisher and Wright surely bears this conclusion out (Provine 1986; Skipper 2002, 2009).

So, DOA does not accord with the early history of evolutionary genetics, despite claims to the contrary (Walsh 2007). This is not necessarily problematic; terms do change over time. But it's worth paying attention to the origins, nonetheless; often terms retain shades of the original meanings for many practitioners, even when these are not explicit. And changes in terms may not have been for the better; sometimes the original definition is the clearest and most consistent. This seems to be true of many of Darwin's definitions, for example. But what we want to suggest here is that not only must we pay attention to the first mentions of a term, but also, we must consider the contemporary uses of the term (as distinguished from biologists' stated definitions that we discussed earlier). As we will show, DOA does not accord with contemporary usage either.

It is important to keep in mind that in the history of population genetics modeling, drift models have played an important role as simplified cases - cases that are mathematically tractable but not thought to correspond to natural systems of alleles. For modelers in stochastic population genetics, starting with the simplest mathematical case and adding complexity is common practice. Indeed in their now classic, $A n$ Introduction to Population Genetics Theory, James Crow and Motoo Kimura explicitly structure their chapter on 
stochastic processes with the simplest case of drift at the beginning and fluctuating selection intensities at the end (Crow and Kimura 1970). However, with the rise of Molecular Population Genetics in the 1960s, these mathematically tractable drift models were transformed from simplest cases to descriptions of real processes describing real systems of amino acid and nucleotide substitutions.

Consider the specific case of the Infinite Alleles Model created by Crow and Kimura in the early 1960s (Kimura and Crow 1964). Crow and Kimura created the Infinite Alleles model because they wanted to address the proportion of overdominant loci that could be maintained in a population (Crow 1989; Dietrich 1994, 2006). Their goal was to argue against advocates of the Balance Position in evolutionary genetics who claimed that large numbers of heterozygous combinations were maintained in natural populations. To model this situation, Crow first assumed that every mutation was a mutation to a new allele (hence the nearly infinite number of alleles that give the model its name). Then Kimura first solved the diffusion equations for the case of neutrality, where there was no selection or $s=0$. This model was then made more complex by adding selection, but even then simplifying assumptions about selective values were made in order to make "the mathematics more manageable" (Kimura and Crow 1964: 728). Kimura and Crow note that this model is "unrealistic," yet they use it because in their words, "It can help provide some insight as to what situations are possible or likely in a natural population." More specifically, the case of drift establishes the maximum heterozygosity per locus and provides a point of contrast for the effects of different forms of selection on heterozygosity. Nowhere in this paper do Kimura and Crow argue that there are neutral alleles in nature or that drift is a predominant force in nature. By 1968, however, Kimura had become convinced that this simplest mathematical case was in fact representative of the observed polymorphisms in natural populations. Kimura was not moved to reify neutral alleles and drift based on their role in stochastic population genetics models. Rather this shift was motivated by a variety of evidence from comparative biochemistry, including data on rate of molecular change, which would create an intolerable genetic load unless many mutants were neutral, the redundancy of the genetic code, which created synonymous mutations with no known selective effect, the constant molecular rate of change, which could not be explained by selection, to name only a few of the arguments. While the neutral theory was controversial, the point here is that the decision to advocate random genetic drift as a significant, naturally occurring biological process was supported by multiple lines of evidence, independent of how those processes were modeled or compared to selective processes.

In his general discussions of drift and stochastic population genetics models, Kimura clearly indicates that he believes that the patterns of randomly changing gene frequencies are the result of either a process of random gamete sampling or a process of the random fluctuation of selection intensities (Kimura 1964, Crow and Kimura 1964). In doing so, he places himself in a tradition of modeling that he traces to Wright. One way of thinking about Kimura's decision to advocate the neutral theory is that he believed that he had new physical evidence for the importance of the process of random gamete sampling on changes in molecular sequences. DOA fails to account for the way Kimura arrived at this conclusion; in particular, it fails to account for Kimura's shift to a realist's understanding of his drift models. It also fails to account for Kimura's use of biochemical data as evidence for indiscriminate sampling; if drift were only deviation from selection expectations, this evidence would be irrelevant and unneeded.

In short, neither the early nor the more recent history of population genetics supports the DOA view. Indeed, DOA is not only inconsistent with original and more recent conceptions of drift, DOA distorts how biologists in practice have reasoned about the process. In addition, the discussion in the present section amplifies our argument of the previous section - mathematical models are not the ultimate resource in understanding biological concepts, particularly drift.

\section{Conclusion}

Our conclusions pertain to the interpretation of models in general as well as the interpretation of drift models in particular. With respect to the interpretation of models in general, we have used the case of drift to try to show that when one is interpreting models, it is a mistake to rely solely on scientists' and philosophers' stated definitions (in any case, both scientists and philosophers often disagree amongst themselves) and a mistake to rely solely on mathematics, the latter mistake being the one that proponents of 
DOA seem to commit. In particular, examination of the mathematics alone cannot tell us whether the terms referred to in the model are purely mathematical or whether they represent physical processes. Instead, we have argued that when interpreting mathematical models, one needs to take into account the original purpose for which the model was developed (what it was taken to represent) as well as its contemporary uses, together with criteria such as clarity and consistency. Our position on interpreting mathematical models echoes views articulated by other philosophers of science such as Ron Giere, Paul Humphreys and others.

With respect to the interpretation of drift models in particular, we have argued that the DOA view of drift, with its reliance on mathematical models alone, fails to yield a proper conceptualization of drift. We have tried to establish this conclusion by showing how simply relying on models can take us down wrong interpretive paths and by examining historical and contemporary biological practice. Indeed, we believe that our argument goes some distance toward establishing the positive claim that drift is best understood by looking not at mathematical models alone, but at those models in relation to the physical processes being modeled, namely the various indiscriminate sampling processes.

When one takes first usage and contemporary biological practice into account, starting with the motivation for Wright's and Fisher's early drift models and ending with Kimura's neutral theory, a very different picture of the concept of drift emerges. On this view, drift is a term designating a set of physical processes, arguably, indiscriminate sampling processes (Beatty 1984; Hodge 1987; Millstein 2002, 2005; Millstein and Skipper 2007, Dietrich and Millstein 2008). Drift is not a purely mathematical outcome.

\section{Literature Gited}

Beatty, J. 1984. Chance and Natural Selection. Philosophy of Science 51:183-211.

Beatty, J. 1992. Random Drift. In Keywords in Evolutionary Biology, edited by EF Keller and EA Lloyd. Harvard University Press.

Brandon, R. 2005. The Difference Between Selection and Drift: A Reply to Millstein. Biology \& Philosophy 20 (1):153-170.

Cain, AJ and JD Currey. 1963. Area Effects in Cepaea. Philosophical Transactions of the Royal Society of London B 246:1-81.

Crow, JF 1989. Twenty-Five Years Ago in Genetics: The Infinite Alleles Model. Genetics 121:631-634.

Crow, JF and M Kimura. 1970. An Introduction to Population Genetics Theory. Harper \& Row Publishers.

Dietrich, MR. 1994. The Origins of the Neutral Theory of Molecular Evolution. Journal of the History of Biology 27:21-59.

Dietrich, MR. 2006. Three Perspectives on Neutrality and Drift in Molecular Evolution. Philosophy of Science 73:666-677.

Dietrich, MR. and RL Millstein. 2008. The Role of Causal Processes in the Neutral and Nearly Neutral Theories. Philosophy of Science 75 (5):548-559.

Dietrich, MR. and RA Skipper Jr. 2007. Manipulating Underdetermination in Scientific Controversy: The Case of the Molecular Clock. Perspectives on Science 15:295-326.

Ewens, WJ. 2004. Mathematical Population Genetics. Second ed. Springer-Verlag.

Fisher, RA. 1921. Review of The Relative Value of the Processes Causing Evolution. The Eugenics Review 13:467-470.

Fisher, RA. 1922. Darwinian Evolution of Mutations. Eugenics Review 14:31-34.

Fisher, RA. 1922. On the Dominance Ratio. Proceedings of the Royal Society of Edinburgh 42:321-341.

Fisher, RA. 1947. The Spread of a Gene in Natural Conditions in a Colony of the Moth Panaxia Dominula L. Heredity 1 (II): 143-174.

Futuyma, D J. 2005. Evolution. Sinauer \& Associates.

Giere, RN. 1988. Explaining Science: A Cognitive Approach. University of Chicago Press.

Gillespie, JH. 2004. Population Genetics: A Concise Guide. Second ed. Johns Hopkins University Press.

Hagedoorn, AL and AC Hagedoorn. 1921. On the Relative Value of the Processes Causing Evolution. The Hague: Nijhoff.

Hartl, DL and AG Clark. 1996. Principles of Population Genetics. Sinauer \& Associates. 
Hodge, MJS. 1987. Natural Selection as a Causal, Empirical, and Probabilistic Theory. In The Probabilistic Revolution, edited by L. Krüger. MIT Press.

Kimura, M. 1968a. Evolutionary Rate at the Molecular Level. Nature 217:624-626.

Kimura, M. 1968b. Genetic Variability Maintained in a Finite Population Due to Mutational Production of Neutral and Nearly Neutral Isoalleles. Genetical Research 11:247-269.

Kimura, M. 1983. The Neutral Theory of Molecular Evolution. Cambridge University Press.

Kimura, M and JF Crow. 1964. The Number of Alleles that Can Be Maintained in a Finite Population. Genetics 49:725-738.

King, JL and TH Jukes. 1969. Non-Darwinian Evolution. Science 164:788-798.

Kreitman, M. 2000. Methods to Detect Selection in Populations with Application to the Human. Annual Review of Genomic and Human Genetics 1:539-559.

Lewontin, RC. 1963. Models, Mathematics, and Metaphors. Synthese 15:222-224.

Lloyd, EA. 1988. The Structure and Confirmation of Evolutionary Theory. Greenwood Press.

Matthen, M and A Ariew. 2002. Two Ways of Thinking About Fitness and Natural Selection. The Journal of Philosophy 99:55-83.

Mills, SK and J Beatty. 1979. The Propensity Interpretation of Fitness. Philosophy of Science 46 (2): 263-286.

Millstein, RL. 2002. Are Random Drift and Natural Selection Conceptually Distinct? Biology and Philosophy 17 (1):33-53.

Millstein, RL. 2005. Selection vs. Drift: A Response to Brandon's Reply. Biology and Philosophy 20 (1): 171-175.

Millstein, RL and RA Skipper. 2007. Population Genetics. In The Cambridge Companion to the Philosophy of Biology, edited by D. Hull and M. Ruse. Cambridge University Press.

Mitchell, S and MR. Dietrich. 2006. Integration without Unification: An Argument for Pluralism in the Biological Sciences. The American Naturalist 168 (S6):S73-S79.

Pigliucci, M and J Kaplan. 2006. Making Sense of Evolution: The Conceptual Foundations of Evolutionary Biology. University of Chicago Press.

Plutynski, A. 2007. Drift: A Historical and Conceptual Overview. Biological Theory 2 (2):156-167.

Provine, WB. 1986. Sewall Wright and Evolutionary Biology. University of Chicago Press.

Richardson, RG. 2006. Chance and the Patterns of Drift: A Natural Experiment. Philosophy of Science 73:642-654.

Ridley, M. 2003. Evolution. Third ed. Blackwell.

Roughgarden, J. 1996. Theory of Population Genetics and Evolutionary Ecology: An Introduction. Prentice Hall.

Skipper, Jr., RA. 2000. The R.A. Fisher-Sewall Wright Controversy in Philosophical Focus: Theory Evaluation in Population Genetics. PhD Dissertation. Committee on the History and Philosophy of Science, University of Maryland, College Park.

Skipper, Jr., RA. 2002. The Persistence of the R. A. Fisher-Sewall Wright Controversy. Biology and Philosophy 17:341-367.

Skipper, Jr., RA. 2006. Stochastic Evolutionary Dynamics: Drift vs. Draft. Philosophy of Science 73:655-665.

Skipper, Jr., RA. 2009. Revisiting the Fisher-Wright Controversy. In Descended from Darwin: Insights into the History of Evolutionary Studies, 1900-1970, edited by J. Cain and M. Ruse. American Philosophical Society.

Walsh, DM. 2007. The Pomp of Superfluous Causes: The Interpretation of Evolutionary Theory. Philosophy of Science 74:281-303.

Walsh, D M., T Lewens and A Ariew. 2002. The Trials of Life: Natural Selection and Random Drift. Philosophy of Science 69:452-473.

Wright, S. 1930. The Genetical Theory of Natural Selection: A Review. The Journal of Heredity 21:349-356.

Wright, S. 1931. Evolution in Mendelian Populations. Genetics 16:97-159. 
Wright, S. 1932. The Roles of Mutation, Inbreeding, Crossbreeding and Selection in Evolution. Proceedings of the Sixth International Congress of Genetics 1:356-366.

Wright, S. 1937. The Distribution of Gene Frequencies in Populations. Proceedings of the National Academy of Sciences of the United States of America 23 (6):307-320.

Wright, S. 1948. On the Roles of Directed and Random Changes in Gene Frequency in the Genetics of Populations. Evolution 2 (4):279-294.

Wright, S. 1951. Fisher and Ford on the 'Sewall Wright Effect'. American Scientist 39:452-458, 479.

Wright, S. 1955. Classification of the Factors of Evolution. Cold Spring Harbor Symposia on Quantitative Biology 20:16-24.

\section{NOTES}

(1) Authors often use the term "random sampling" rather than "indiscriminate sampling," but given the many connotations of the word "random", we think "indiscriminate" better captures the intended meaning. We will use this interpretation throughout the subsequent discussion.

(2) Pigliucci and Kaplan (personal communication) say that they did not intend to endorse DOA, but rather that they think that drift is caused by indiscriminate sampling as well as various random events at the level of individuals.. Their view is different from DOA, they contend, in that they think "drift" can refer either to the difference between the actual changes in a population and those changes that would be predicted from the differences in fitness or to the degree of 'scatter' around the (expected) mean changes in the population (the shape and size of the distribution). Perhaps this might be seen as a variant of DOA.

(3) As suggested by our discussion above, our view is that such readings are highly selective, ignoring discussions of indiscriminate sampling.

(4) The range of standards used in the evaluation of models and theories is presented in Skipper (2000) and Dietrich and Skipper (2008).

(5) Ewens (2004) makes a similar point.

(6) Of course, what they famously disagreed about was the type and extent of the role of drift, among other things.

(7) Selection, of course, has similarly been described as occurring at many levels of organization.

\section{AGKNOWLEDGMENTS}

We would like to thank Frédéric Bouchard, Jim Griesemer, Jonathan Kaplan, Massimo Pigliucci, Joan Roughgarden, the anonymous referees, and members of the audience at PSA 2008 for helpful comments and/or discussion.

Copyright (C) 2009 Author(s).

This is an open-access article distributed under the terms of the Creative Commons Attribution-NonCommercial-NoDerivs license, which permits anyone to download, copy, distribute, or display the full text without asking for permission, provided that the creator(s) are given full credit, no derivative works are created, and the work is not used for commercial purposes.

ISSN 1949-0739 